\title{
Elastic and anelastic properties close to the Curie temperature of Fe-based bulk metallic glass
}

J. Torrens-Serra, M. Stoica, J. Bednarcik, J. Eckert, and S. Kustov

Citation: Appl. Phys. Lett. 102, 041904 (2013); doi: 10.1063/1.4789776

View online: https://doi.org/10.1063/1.4789776

View Table of Contents: http://aip.scitation.org/toc/apl/102/4

Published by the American Institute of Physics

\section{Articles you may be interested in}

Fe-based bulk metallic glasses: Brittle or ductile?

Applied Physics Letters 105, 161901 (2014); 10.1063/1.4899124

Cr-based bulk metallic glasses with ultrahigh hardness

Applied Physics Letters 106, 251905 (2015); 10.1063/1.4923210

Tough Fe-based bulk metallic glasses

Applied Physics Letters 92, 091918 (2008); 10.1063/1.2890489

Critical cooling rate and thermal stability of $\mathrm{Zr}-\mathrm{Ti}-\mathrm{Cu}-\mathrm{Ni}-\mathrm{Be}$ alloys

Applied Physics Letters 78, 1213 (2001); 10.1063/1.1350624

The viscosity of the $\mathrm{Zr}_{46.75} \mathrm{Ti}_{8.25} \mathrm{Cu}_{7.5} \mathrm{Ni}_{10} \mathrm{Be}_{27.5}$ bulk metallic glass forming alloy in the supercooled liquid Applied Physics Letters 67, 3260 (1995); 10.1063/1.114891

Ductile $\mathrm{Fe}-\mathrm{Nb}-\mathrm{B}$ bulk metallic glass with ultrahigh strength

Applied Physics Letters 92, 251906 (2008); 10.1063/1.2949747

\section{Instruments for Advanced Science}

Contact Hiden Analytical for further details: w www.HidenAnalytical.com E info@hiden.co.uk CLICK TO VIEW our product catalogue

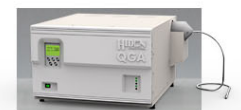

Gas Analysis - dynamic measurement of reaction gasstreams cataysis and thermal analysis molecular beam studes dissolved species probes fermentation, envirormental and ecological studis:

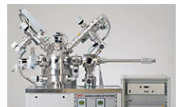

Surface Science UHVTPD

, end point detection in in beam etch , elemental imaging-surface mapping

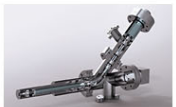

Plasma Diagnostics , plasma source characterization etch and deposition process reaction kinetic studies analysis of neutral and radical species

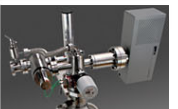

Vacuum Analysis partial pressure measurement and control of process gases , reactive sputter process control vacuum diagnostic 


\title{
Elastic and anelastic properties close to the Curie temperature of Fe-based bulk metallic glass
}

\author{
J. Torrens-Serra, ${ }^{1,2}$ M. Stoica, ${ }^{2}$ J. Bednarcik, ${ }^{3}$ J. Eckert, ${ }^{2,4}$ and S. Kustov ${ }^{1}$ \\ ${ }^{1}$ Departament de Física, Universitat de les Illes Balears, Cra. Valldemossa km 7.5, E-07122 Palma de \\ Mallorca, Spain \\ ${ }^{2} I F W$-Dresden, Institut für Komplexe Materialien, Helmholtzstr. 20, D-01069 Dresden, Germany \\ ${ }^{3}$ Deutsches Elektronen-Synchrotron DESY, Notkestr. 85, D-22607 Hamburg, Germany \\ ${ }^{4}$ TU Dresden, Institut für Werkstoffwissenschaft, Helmholtzstr. 7, D-01069 Dresden, Germany
}

(Received 10 December 2012; accepted 15 January 2013; published online 28 January 2013)

\begin{abstract}
Linear and non-linear ultrasonic damping, Young's modulus, and non-linear modulus defect have been studied in a Fe-based bulk metallic glass close to its Curie temperature, $T_{C}$. We found that the acoustic technique used offers four different parameters which can be used to evaluate the $T_{C}$. We show that ultrasonic measurements at a frequency of around $10^{5} \mathrm{~Hz}$ detect both critical phenomena close to $T_{C}$ and intrinsic magnetomechanical damping in the ferromagnetic state. However, the values of absorption and Young's modulus anomaly observed over the critical range are quite low and do not permit reliable analysis of the critical exponents. (C) 2013 American Institute of Physics. [http://dx.doi.org/10.1063/1.4789776]
\end{abstract}

Ferromagnetic bulk metallic glasses (BMG) are promising materials due to their very good soft magnetic properties, ultrahigh fracture strength, and good corrosion resistance..$^{1-3}$ The principal characteristics to serve as soft magnetic alloys $\operatorname{are}^{4}$ (1) high saturation magnetization, (2) high permeability, (3) low coercivity, (4) low magnetostriction, and (5) high Curie temperature. Most of Fe-based bulk metallic glasses developed in the past possess all these properties. Except for the coercive force, the magnetic properties are directly related to the composition of the alloy or, more precisely, to the relation between the ferromagnetically and antiferromagnetically coupled atom pairs. The Curie temperature, a crucial parameter from both a practical and a fundamental point of view, is very sensitive to variations of the composition of alloys and can be tuned this way. The possibility to control the Curie temperature in $\mathrm{BMG}$, on the other hand, requires a clear algorithm of its monitoring. This temperature is commonly determined by measuring the magnetization during a continuous temperature sweep under an applied magnetic field by means of either a Faraday magnetic balance or a vibrating sample magnetometer. Different interpretations can be found in literature of the dependences obtained this way. The Curie point is evaluated simply from the drop of the magnetization $M(T)$, from the minimum of the $d M / d T$ (Ref. 5) or using more elaborated methods like Herzer fitting $^{6}$ or Arrott plots. ${ }^{7}$ The aforementioned methods imply application of an external field which strongly affects the otherwise disordered state of magnetic moments in the vicinity of $T_{C}$. This approach has recently been critically assessed $^{5}$ and a conclusion has been drawn that even the most elaborated techniques ${ }^{7,8}$ do not provide reliable values of the "true" intrinsic Curie temperature, the estimation of which is notably sensitive even to the shape of the sample through the demagnetizing factor. A method to identify the Curie point from synchrotron hard $\mathrm{x}$-ray diffraction has recently been suggested. ${ }^{9}$ The method is based in the fact that the thermal expansion coefficient in Fe-based metallic glasses depends on the magnetic state of the alloy. Thus, measuring a structural property a magnetic one can be determined. However, the method ${ }^{9}$ requires a special synchrotron $\mathrm{x}$-ray source, which does not seem to be practical. On the other hand, other structural properties may also change upon long-range magnetic ordering of the BMG. It is well known that elastic and magnetic properties are related through magnetoelastic coupling. Thus, similar to the case of crystalline materials, expected variations of the elastic constants can serve as a simple method of monitoring the Curie temperature of BMG. Apart from the verification of this practical issue, studying elastic and anelastic effects accompanying ferromagnetic ordering in BMG is of interest since they might characterize both the critical spin fluctuations during ferromagnetic ordering and the formation of magnetic domain structure below the $T_{C}$ temperature.

More specifically, it is known that ferromagnets demonstrate a number of anelastic effects, referred to as magnetomechanical damping (MMD). ${ }^{10}$ Traditionally, three ingredients of MMD are distinguished: linear micro- and macro-eddy currents and non-linear hysteretic components. From the point of view of domain structure characterization, the most informative are the first and the last components, which are supposed to reflect small scale linear and large scale non-linear oscillations, respectively, of non $180^{\circ}$ domain walls. ${ }^{10}$ MMD is supposed to exist over the entire temperature range of ferromagnetic state, below $T_{C}$. If the Curie temperature is understood as a temperature beyond which the long-range magnetic ordering (magnetic domains) is absent, the non-linear MMD should vanish at this point. Metallic glasses are perfect objects to apply this method, since they do not contain other sources of the non-linear internal friction as dislocations or twin boundaries. Thus, the nonlinear internal friction is expected to be of purely magnetic origin.

So far, elastic properties of BMG have been studied intensively mostly at elevated temperatures, see, e.g., recent review, ${ }^{11}$ and anelasticity of BMG - in the range of structural relaxation in non-magnetic alloys. ${ }^{12-15}$ Only a few 
publications dealt with the MMD in amorphous alloys in a form of ribbons ${ }^{16-21}$ and thin wires. ${ }^{22}$ The interest has been focused on the effect of strain amplitude and polarizing field on MMD and $\triangle \mathrm{E}$ effect at ambient temperature, leaving the elastic and anelastic properties of BMG close to the magnetic ordering temperature essentially unexplored.

Acoustic methods are also traditionally used to characterize critical phenomena which occur in the vicinity of the ordering temperature of various metallic and insulator compounds into ferro- and antiferromagnetic states. ${ }^{23}$ Acoustic experiments are usually performed by the pulse-echo technique for frequencies exceeding $10 \mathrm{MHz}$. However, intrinsic limitations on the size of BMG impose severe restrictions on application of this method in the case of amorphous ferromagnets.

The aim of the present work was, using measurements at an ultrasonic frequency of around $10^{5} \mathrm{~Hz}$,

- to demonstrate that studying elastic and anelastic properties is a sensitive method to detect, by monitoring several possible indicators, magnetic ordering in BMG without affecting this ordering by polarizing field;

- to analyze the behavior of elastic and anelastic properties of BMG over the critical range of magnetic ordering.

The $\mathrm{Fe}_{61} \mathrm{Cr}_{4} \mathrm{Mo}_{14} \mathrm{C}_{15} \mathrm{~B}_{6}$ alloy has been chosen as test material due to its good glass forming ability. ${ }^{24-27}$ The BMG were produced by direct mold casting in the form of $1.5 \mathrm{~mm}$ diameter rods. The amorphous state of the rods has been checked by high-energy X-ray diffraction with a beam energy $100 \mathrm{keV}(\lambda=0.123984 \AA)$ at the wiggler beamline BW5 of the positron storage ring DORIS III (Hamburg, Germany). The rods have been scanned along its length in steps of $2 \mathrm{~mm}$, all of them exhibiting the typical broad halo (not shown here). The elastic (Young's modulus, YM) and anelastic properties (internal friction represented by the logarithmic decrement, $\delta$ ) were studied using longitudinal resonant oscillations of bar-shaped samples with a length $l=28.3 \mathrm{~mm}$ at a frequency close to $90 \mathrm{kHz}$. The technique has been described in detail elsewhere. ${ }^{28}$ In the present experiments, the internal friction was measured as a function of temperature, quasi simultaneously for two different amplitudes, the low amplitude falling within the linear range $\left(\varepsilon_{l a}=2 \times 10^{-7}\right)$ and the high one in the non-linear regime $\left(\varepsilon_{h a}=1 \times 10^{-5}\right)$, the method being described previously, see, e.g., Ref. 29. The amplitude-dependent component of the internal friction, ADIF, $\delta_{\mathrm{h}}$, was obtained as the difference of the internal friction values measured at $1 \times 10^{-5}\left(\delta_{h a}\right)$ and $2 \times 10^{-7}\left(\delta_{l a}\right)$,

$$
\delta_{h}=\delta_{h a}-\delta_{l a} .
$$

The Young's modulus was determined from the resonant frequency $f$ of the fundamental mode of longitudinal oscillations of samples: $E=4 \rho l^{2} f^{2}$, where $\rho$ is the density of the alloy. The non-linear (strain amplitude-dependent) Young's modulus defect (ADMD) was calculated as

$$
\left.\frac{\Delta E}{E}\right|_{h}=\frac{E_{l a}-E_{h a}}{E_{l a}}
$$

where $E_{l a}$ and $E_{h a}$ are the YM values determined for low and high strain amplitude, respectively.
Temperature dependences were measured in an Oxford close-loop cryostat with a temperature rate of around $0.5 \mathrm{~K} /$ min. Oscillators consisting of quartz transducers with attached samples were placed into a copper chamber containing $\mathrm{He}$ atmosphere under pressure of around $0.3 \mathrm{~atm}$. The temperature inside the chamber was registered by means of an additional calibrated Lakeshore Cernox resistive sensor (the uncertainty of calibration and polynomial fitting does not exceed $0.1 \mathrm{~K}$ over the temperature range studied) placed in a close vicinity (within $2-3 \mathrm{~mm}$ distance) from the sample. The good correspondence between the temperature of the oscillator and the registered one was verified by controlling the temperature of $\mathrm{Na}$ relaxation peak ${ }^{30}$ at around $39 \mathrm{~K}$ in quartz transducers.

To interpret the data of acoustic measurements, preliminary experiments were carried out to determine the Curie temperature from the imaginary part $(X)$ of the AC impedance $(Z)$ measured as a function of temperature at $686 \mathrm{~Hz}$ by conventional four probe technique. The magnetic permeability $(\mu)$ is related to the imaginary part of the impedance of a cylindrical conductor at low enough frequency (neglecting the skin-effect) in a following simple way:

$$
X=\frac{\omega \mu l}{8 \pi},
$$

with $\omega$ the angular frequency and $l$ the length of the sample. The imaginary part of $\mathrm{AC}$ impedance on cooling against temperature is plotted in Fig. 1. The curve shows the typical behavior for a para-ferromagnetic transition with an asymmetric Hopkinson peak - indication of the formation of magnetic domains and emergence of magnetic anisotropy. The Curie temperature is determined from this curve as the intersection between the tangent at the inflexion point of the curve and the horizontal line representing paramagnetic $X$ value, yielding $T_{C}=160.3 \mathrm{~K}$.

Figure 2(a) shows the internal friction registered at two different amplitudes versus temperature on cooling. In the paramagnetic state, the curves at low and high strain amplitudes coincide, indicating, according to Eq. (1), the absence

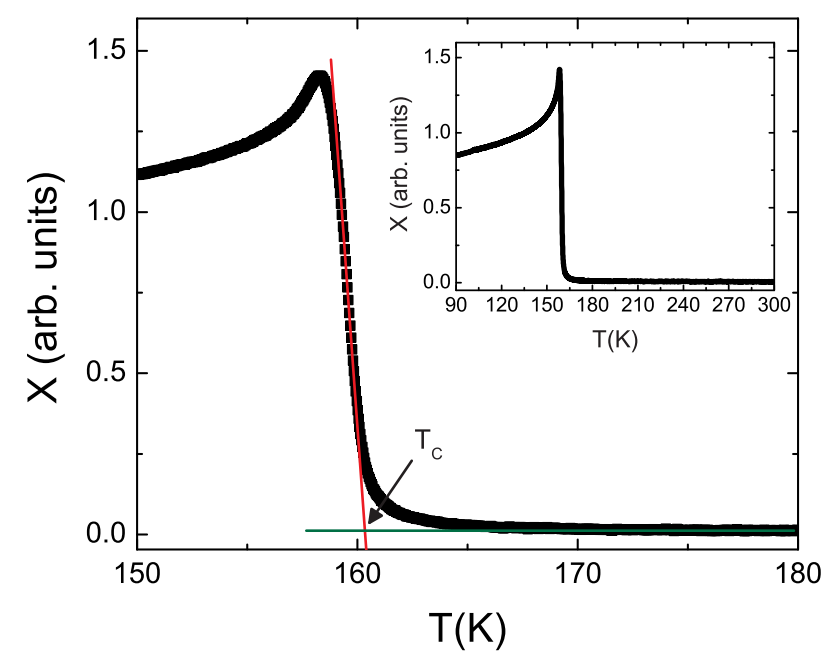

FIG. 1. Temperature dependence of the imaginary part of AC impedance $(X)$ in the vicinity of para-ferromagnetic transition for a sample of $\mathrm{Fe}_{61} \mathrm{Cr}_{4}$ $\mathrm{Mo}_{14} \mathrm{C}_{15} \mathrm{~B}_{6}$ bulk metallic glass. Measurements during cooling at $0.5 \mathrm{~K} / \mathrm{min}$. Inset: general view of the temperature spectra between 90 and $300 \mathrm{~K}$. 


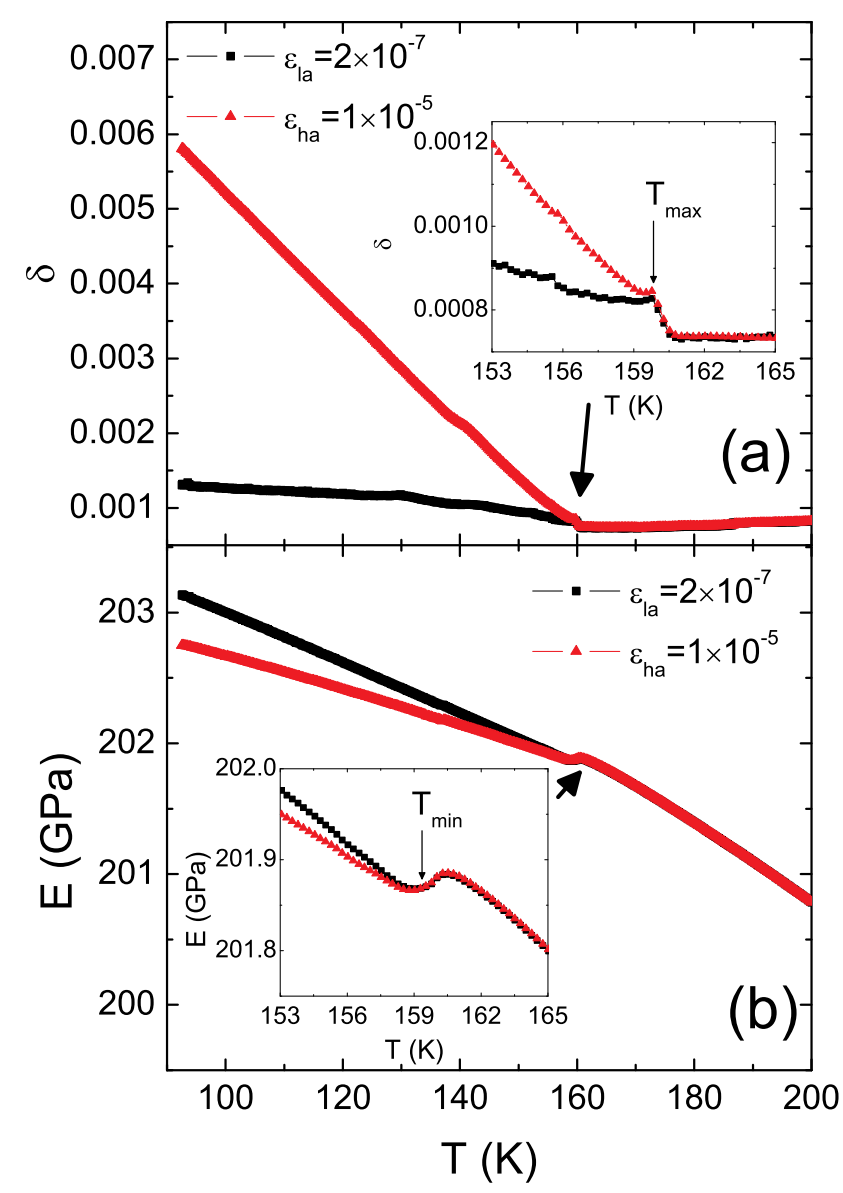

FIG. 2. Temperature dependence of the internal friction (a) and Young's modulus (b) for a sample of $\mathrm{Fe}_{61} \mathrm{Cr}_{4} \mathrm{Mo}_{14} \mathrm{C}_{15} \mathrm{~B}_{6}$ bulk metallic glass measured at two strain amplitudes $\varepsilon_{\mathrm{la}}=2 \times 10^{-7}$ (black squares) and $\varepsilon_{\text {ha }}=1 \times 10^{-5}$ (red triangles) during cooling at $0.5 \mathrm{~K} / \mathrm{min}$. Inset: details of the curves close to the Curie temperature.

of the non-linear component in the total internal friction. As mentioned above, this is expected since such traditional source of the ADIF as dislocations do not exist in BMG. Below approximately $161 \mathrm{~K}$, a synchronous rapid increase of both the low- and high-amplitude internal friction values is observed. The inset in Fig. 2(a) shows that the lowamplitude internal friction reaches a maximum at around $T_{\text {max }}=159.8 \mathrm{~K}$ and then falls off slightly. Just at the temperature of the maximum, the low- and high-amplitude curves diverge, indicating that the non-linear MMD emerges at this point.

Figure 2(b) depicts the YM temperature dependence corresponding to the internal friction data in Fig. 2(a). Similar to the data in Fig. 2(a), no non-linear effects are detected above approximately $160 \mathrm{~K}$. The YM registered at high and low strain amplitudes demonstrates a minimum at around $T_{\min }=159.5 \mathrm{~K}$. The low- and high-amplitude curves diverge starting approximately from this temperature, reflecting, similar to the internal friction data, the emergence of the nonlinear anelastic contribution due to the hysteretic motion of magnetic domain walls. Thus, both the $T_{\max }$ and $T_{\min }$ are rather close to the $T_{C}$ temperature derived from the $\mathrm{AC}$ impedance data.

The existence of an ultrasonic internal friction maximum concomitant with the YM (or sound velocity) minimum close to the Curie temperature is a hallmark of critical spin fluctuation phenomenon, ${ }^{23}$ affecting acoustic oscillations through magnetoelastic coupling. The precision of the present measurements does not permit us to analyze possible difference in the temperatures of observed internal friction maximum and modulus minimum around $T_{C}$. Moreover, strictly speaking, the temperatures of these extrema should be compared after subtracting the corresponding background temperature dependences, which does not seem to be reliable in view of the small effects detected. Nevertheless, it should be mentioned that, according to the detailed studies of the critical sound absorption in different materials (see, e.g., Refs. 31, 32, and references therein), the critical absorption maximum is slightly (of the order of $0.01-0.1 \mathrm{~K}$ ) displaced to higher temperature with respect to the YM modulus. According to Ref. 32, both extrema in Gd are situated somewhat (also about $0.1 \mathrm{~K}$ ) below the Curie temperature, determined by the kink-method. These regularities seem to be qualitatively reproduced in the present BMG. At the same time, the values of the internal friction maximum observed in BMG at $90 \mathrm{kHz}$ ( $\delta_{\max }$ below $10^{-4}$ after subtracting the background) are more than two orders of magnitude lower than the critical attenuation typical in rare earth metals (which are the most extensively investigated) registered for frequencies above 10 MHz, see, e.g., Refs. 31-33. This difference is, likely, at least partly due to the relatively low frequency employed in the present study, since in the ultrasonic $\mathrm{kHz}$ range, the critical attenuation reduces to a quadratic function of frequency. ${ }^{23}$ Nevertheless, it is worthwhile to emphasize that the magnitude of the internal friction peak registered close to $T_{C}$ at a frequency of around $10^{3} \mathrm{~Hz}$ in single crystalline $\mathrm{Gd}$ with stress oriented along the c-axis ${ }^{34}$ is more than an order of magnitude higher than the values obtained in the present work. Moreover, the Young's modulus anomaly over the range of critical fluctuations found in the present BMG corresponds to the sound velocity decrease $\Delta v / v \approx 10^{-4}$, which is 5-10 times lower than, for example, a very similar anomaly observed also at a frequency of around $10^{5} \mathrm{~Hz}$ during antiferromagnetic ordering in $\mathrm{Y}_{3} \mathrm{Fe}_{5} \mathrm{O}_{12}$ single crystals ${ }^{35}$ and at a frequency of around $10^{3} \mathrm{~Hz}$ in $\mathrm{Gd} .^{34,36}$ Therefore, weak critical sound absorption and Young's modulus anomaly close to the Curie temperature in the present BMG should be related to its weak spin-phonon interaction compared to crystalline solids. It remains to be seen whether or not weak acoustic anomalies over the range of critical spin fluctuations is a generic property of amorphous ferromagnets, probably due to the lack of long-range structural periodicity. The short-range atomic ordering in BMG allows propagation of only highly damped phonon modes.

The non-linear anelastic effects emerging close to the $T_{C}$ temperature deserve several comments. Figures 3(a) and 3(b) demonstrate temperature scans of the ADIF and of the ADMD. The insets in Figs. 3(a) and 3(b) show the details of the ADIF and ADMD behavior close to $T_{C}$. As mentioned before, a remarkable property of BMG is the absence of the non-linear anelastic effects in the paramagnetic phase due to the absence of dislocations. The non-linear anelasticity in the ordered state has purely magnetic origin, related to the hysteretic motion of domain walls. The characteristic temperatures of the emergence of the non-linear ultrasound absorption and of the non-linear YM defect of the magnetic 


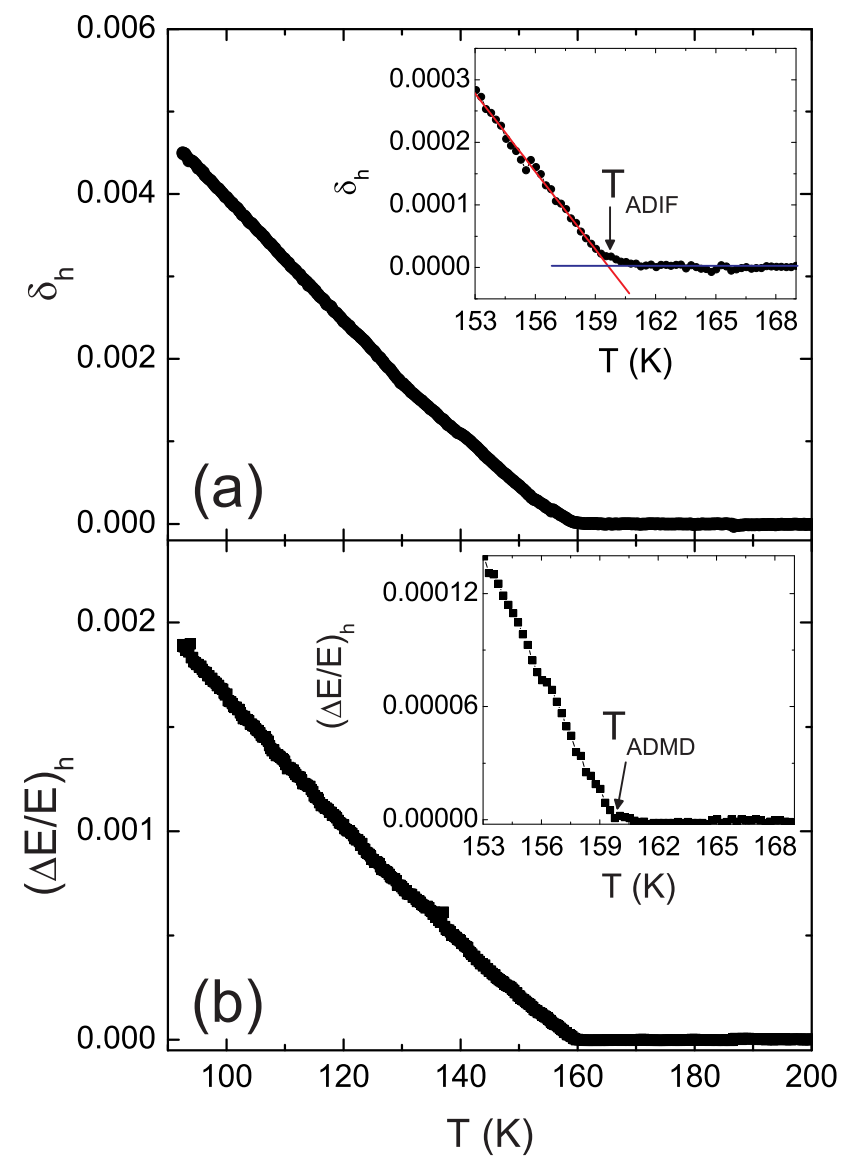

FIG. 3. Temperature dependence of the non-linear component of the internal friction (a) and Young's modulus defect (b) for a sample of $\mathrm{Fe}_{61} \mathrm{Cr}_{4}$ $\mathrm{Mo}_{14} \mathrm{C}_{15} \mathrm{~B}_{6}$ bulk metallic glass during cooling at $0.5 \mathrm{~K} / \mathrm{min}$. The insets show details of the dependences close to the Curie temperature.

origin, $T_{A D I F}$ and $T_{A D M D}$, derived as shown in insets in Figs. 3 (a) and 3(b) yield the same values of $159.8 \mathrm{~K}$ as the internal friction peak in Fig. 2(a), $T_{\max }$. This value is close to the temperature of the YM minimum in Fig. 2(b), $T_{\text {min }}$, and, hence, all four characteristic temperatures determined from the acoustic measurements, $T_{\max }, T_{\min }, T_{A D M D}, T_{A D I F}$ are close to the Curie temperature determined from the AC impedance. Perhaps the interpretation of the $T_{A D M D}$ and $T_{A D I F}$ temperatures is the most straightforward and, in addition, the derivation of these temperatures does not require ambiguous treatment of experimental data, like background subtraction.

In summary, the acoustic technique employed offers 4 different parameters which can be used to evaluate the Curie temperature.

We found that measurements at a frequency of around $10^{5} \mathrm{~Hz}$ allow detecting critical phenomena during ferromagnetic ordering of the Fe-based bulk metallic glass. However, the values of critical absorption and Young's modulus anomaly observed are quite low and point to a weak magnetoelastic coupling in the present bulk metallic glass. It is challenging to claim that weak acoustic anomalies in the range of critical spin fluctuations is a generic property of amorphous ferromagnets, related to the existence of only short range atomic ordering and, hence, only highly damped phonon modes.

J.T.-S. acknowledges the financial support from the Generalitat de Catalunya through the 2009 BP-A 00138 Grant during his postdoctoral stay at IFW-Dresden. J.T.-S. and S.K. acknowledge support from the Spanish Ministerio de Economía y Competitividad, Project MAT2011-28217-C02-01. M. Stoica acknowledges the STO 873/2-1 DFG Grant.

${ }^{1}$ A. Inoue and A. Takeuchi, Acta Mater. 59, 2243 (2011).

${ }^{2}$ A. Inoue, B. L. Shen, and C. T. Chang, Acta Mater. 52, 4093 (2004).

${ }^{3}$ S. Roth, M. Stoica, J. Degmová, U. Gaitzsch, J. Eckert, and L. Schultz, J. Magn. Magn. Mater. 304, 192 (2006).

${ }^{4}$ M. E. McHenry, M. A. Willard, and D. E. Laughlin, Prog. Mater. Sci. 44, 291 (1999).

${ }^{5}$ V. I. Zverev, R. R. Gimaev, A. M. Tishin, Ya. Mudryk, K. A. Gschneidner, Jr., and V. K. Pecharsky, J. Magn. Magn. Mater. 323, 2453 (2011).

${ }^{6}$ G. Herzer, IEEE Trans. Magn. 25, 3327 (1989).

${ }^{7}$ A. Arrott, Phys. Rev. 108, 1394 (1957).

${ }^{8}$ K. P. Belov and A. N. Goryaga, Fiz. Met. Metaloved. II, 3 (1956).

${ }^{9}$ S. Michalik, J. Bednarcik, J. Kovac, H. Franz, and P. Sovak, J. Phys. D: Appl. Phys. 45, 455302 (2012).

${ }^{10}$ J. Degauque, "Magnetic domains," in Mechanical Spectroscopy Q-1 2001 with Application to Materials Science, edited by R. Schaller, G. Fantozzi, and G. Gremaud (Trans. Tech., Zuerich, 2001), pp. 453-482.

${ }^{11}$ W. H. Wang, Prog. Mater. Sci. 57, 487-656 (2012).

${ }^{12}$ J. M. Pelletier, J. Non-Cryst. Solids 325, 133 (2003).

${ }^{13}$ J. M. Pelletier, J. Alloys Compd. 393, 223 (2005).

${ }^{14}$ S. V. Khonik, V. V. Sviridov, O. P. Bobrov, M. Yu. Yazvitsky, and V. A. Khonik, J. Phys.: Condens. Matter 20, 165204 (2008).

${ }^{15}$ J. M. Pelletier, C. Gauthier, J. J. Blandin, and S. Gravier, Solid State Phenom. 184, 393 (2012).

${ }^{16}$ B. S. Berry and W. C. Pritchet, J. Appl. Phys. 47, 3295 (1976).

${ }^{17}$ Y. Y. Kalinin, B. G. Sukhodolov, I. V. Zolotukhin, and V. P. Alyokhin, Fiz. Met. Metalloved. 55, 243 (1983).

${ }^{18}$ N. P. Kobelev, Ya. M. Soifer, V. G. Shteinberg, and Yu. B. Levin, Phys. Status Solidi A 102, 773 (1987).

${ }^{19}$ I. V. Zolotukhin, V. I. Belyavskiy, V. A. Khonik, and T. N. Ryabtseva, Fiz. Met. Metalloved. 68, 185 (1989).

${ }^{20}$ J. Gutiérrez, J. M. Barandiarán, and O. V. Nielsen, Phys. Status Solidi A 111, 279 (1989).

${ }^{21}$ I. V. Zolotukhin, Y. Y. Kalinin, I. V. Sychev, and D. I. Sharshakov, Fiz. Met. Metalloved. 76, 79 (1993).

${ }^{22}$ S. Atalay and P. T. Squire, J. Appl. Phys. 73, 871 (1993).

${ }^{23}$ B. Lüthi, Physical Acoustics in the Solid State (Springer-Verlag, Berlin, 2005).

${ }^{24}$ X. J. Gu, S. J. Poon, and G. J. Shiflet, J. Mater. Res. 22, 344 (2007).

${ }^{25}$ V. Ponnambalam, S. J. Poon, and G. J. Shiflet, J. Mater. Res. 19, 1320 (2004).

${ }^{26}$ Z. P. Lu, C. T. Liu, J. R. Thompson, and W. D. Porter, Phys. Rev. Lett. 92, 245503 (2004).

${ }^{27}$ J. Shen, Q. Chen, J. Sun, H. Fan, and G. Wang, Appl. Phys. Lett. 86, 151907 (2005).

${ }^{28}$ S. Kustov, S. Golyandin, A. Ichino, and G. Gremaud, Mater. Sci. Eng., A 442, 532 (2006).

${ }^{29}$ S. Kustov, S. Golyandin, K. Sapozhnikov, and M. Morin, Scr. Mater. 43, 905 (2000).

${ }^{30}$ D. S. Park and A. S. Nowick, Phys. Status Solidi A 26, 617 (1974).

${ }^{31}$ R. J. Pollina and B. Lüthi, Phys. Rev. 177, 841 (1969).

${ }^{32}$ I. K. Kamilov and Kh. K. Aliev, Phys. Usp. 41, 865 (1998).

${ }^{33}$ B. Lüthi and R. J. Pollina, Phys. Rev. 167, 488 (1968).

${ }^{34}$ Y. I. Spichkin, A. M. Tishin, and K. A. Gschneider, Jr., J. Magn. Magn. Mater. 204, 5 (1999).

${ }^{35}$ Yu. A. Burenkov and S. P. Nikanorov, Mater. Sci. Eng., A 370, 361 (2004).

${ }^{36}$ V. Yu. Bodriakov, A. A. Povzner, and S. A. Nikitin, Eur. Phys. J. B 4, 441 (1998). 